УДК 528.74

\title{
Mathematical Model of the State of Objects Lighting Considering the Terrain
}

\author{
Aleksey I. Tishchenko and Igor V. Lyutikov*b \\ ${ }^{a}$ Military Education and Research Centre of Military-Air Forces \\ «Military-Air Academy \\ Named After Professor N.E. Zhukovsky and Yu.A. Gagarin» \\ 54a Starykh Bolshevikov Str., Voronezh, 394064, Russia \\ ${ }^{b}$ Siberian Federal University \\ 79 Svobodny, Krasnoyarsk, 660041, Russia
}

Received 30.11.2016, received in revised form 24.02.2017, accepted 26.03.2017

This paper presents a mathematical model of the probability of hitting the objects of lightning shields taking into account the terrain with a view to its application to the problems of lightning protection of different objects.

Keywords: lightning protection, area relief, model lightning discharge.

Citation: Tishchenko A.I., Lyutikov I.V. Mathematical model of the state of objects lighting considering the terrain, J. Sib. Fed. Univ. Eng. technol., 2017, 10(4), 467-474. DOI: 10.17516/1999-494X-2017-10-4-467-474.

(c) Siberian Federal University. All rights reserved

* Corresponding author E-mail address: lyutikovigor@mail.ru 


\title{
Математическая модель состояния \\ объектов молниезащиты \\ с учетом рельефа местности
}

\author{
А.И. Тищенко \\ ${ }^{a}$ Военный учебно-научный центр Военно-воздушных сил \\ «Военно-воздушная академия \\ имени профессора Н.Е. Жуковского и Ю.А. Гагарина» \\ Россия, 394064, Воронеж, ул. Старых Большевиков, 54 а \\ ${ }^{6}$ Сибирский федеральный университет \\ Россия, 660041, Красноярск, пр. Свободный, 79
}

Рассмотрена математическая модель вероятности поражения объектов молниезащить с учетом рельефа местности с иелью ее применения к задачам молниезащиты объектов различного назначения

Ключевые слова: молниезащита, рельеф местности, модель грозового разряда.

\section{Введение}

Исследования американских ученых, опубликованные в ноябрьском 2014 г. журнале Science, показывают, что глобальное потепление значительно увеличивает частоту ударов молний. По данным Калифорнийского университета в Беркли, на каждые два удара молнии в 2000 г. придется три удара в 2100-м. Большее число ударов молний сопровождается большим количеством пострадавших людей, вплоть до смертельных исходов.

Средством борьбы с поражением молний объектов инфраструктуры является молниеотвод. Эффективность работы молниеотводов зависит от грамотного их размещения. Оптимизировать размещение средств молниезащиты можно на основе имитационного моделирования процесса протекания разряда молнии и поражения ими объектов инфраструктуры. Одна из таких моделей представлена в работе [1]. Она на основе статистической модели распространения молнии позволяет проводить моделирование стержневой молниезащиты объектов. Модель дает распределения напряженности электростатического поля на объектах молниезащиты и вероятности их поражения молнией с учетом конфигурации защищаемых объектов. В точках с наибольшим значением наводимой напряженности электростатического поля и вероятности поражения объектов молнией рекомендуют устанавливать стержневые мониеотводы.

На развитие кучево-дождевой облачности - источника молний оказывает влияние рельеф местности. Следует ожидать, что на распределение наводимой напряженности электростатического поля на объектах молниезащиты и на вероятности их поражения молнией также будет влиять рельеф местности. Поэтому имитационные модели состояния объектов молниезащиты должны учитывать как особенности конфигурации объектов молниезащиты, так и особенности рельефа местности, на которой расположены эти объекты. 
Целью исследования стала разработка численной модели расчета напряженности электростатического поля и вероятности поражения объектов защиты с учетом рельефа местности.

В настоящей статье решаются следующие задачи:

- разрабатывается математическая модель состояния объектов молниезащины с учетом рельефа местности;

- производится расчет распределений напряженности электростатического поля и вероятности поражения объектов молниезащиты с учетом рельефа местности.

\section{Математическая модель состояния объектов молниезащиты с учетом рельефа местности}

В математической модели объекты защиты и рельеф местности представляются в виде полигонов, которые разбиваются на точки (узлы сетки). Пример простейшего объекта, ангар, изображен на рис. 1.

Каждый узел представляется в виде сферы с зарядом, наводимым внешним полем с некоторой напряжённостью $E_{0}$, созданной кучево-дождевой облачностью с грозовыми явлениями, и радиусом, равным половине шага регулярной сетки $d$. Для полученной системы сфер с центрами в точках $\left(x_{i}, y_{i}, z_{i}\right)(i=1, \ldots, n)$ вычисляют значения зарядов $q_{i}$, используя закон Кулона, принцип суперпозиции и зеркального отражения зарядов, в предположении, что сферы заземлены и имеют нулевой потенциал:

$$
\begin{aligned}
& \frac{1}{d} \frac{1}{2 z_{1}+d} q_{1}+\frac{1}{a_{12}} \frac{1}{b_{12}} q_{2}+\ldots+\frac{1}{a_{1 n}} \frac{1}{b_{1 n}} q_{n}=4 \pi \varepsilon_{0} E_{0} z_{1}, \\
& \frac{1}{a_{21}} \frac{1}{b_{21}} q_{1}+\frac{1}{d} \frac{1}{2 z_{2}+d} q_{2}+\ldots+\frac{1}{a_{2 n}} \frac{1}{b_{2 n}} q_{n}=4 \pi \varepsilon_{0} E_{0} z_{2}, \\
& \ldots \\
& \frac{1}{a_{n 1}} \frac{1}{b_{n 1}} q_{1}+\frac{1}{a_{n 2}} \frac{1}{b_{n 2}} q_{2}+\ldots+\frac{1}{d} \frac{1}{2 z_{n}+d} q_{n}=4 \pi \varepsilon_{0} E_{0} z_{n},
\end{aligned}
$$

где $\varepsilon_{0}$ - диэлектрическая проницаемость воздуха, $x_{m}, y_{m}, z_{m}$ - координаты центра $m$-й сферы; $x_{n}, y_{n}, z_{n}$ - координаты центра $n$-й сферы; $a_{m n}=\sqrt{\left(\begin{array}{ll}x_{m} & x_{n}\end{array}\right)^{2}+\left(\begin{array}{ll}y_{m} & y_{n}\end{array}\right)^{2}+\left(\begin{array}{ll}z_{m} & z_{n}\end{array}\right)^{2}}$ - расстояние

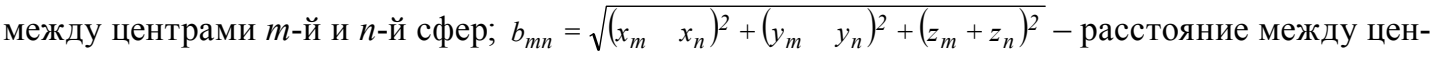
трами $m$-й сферы и зеркального отражения $n$-й сферы.

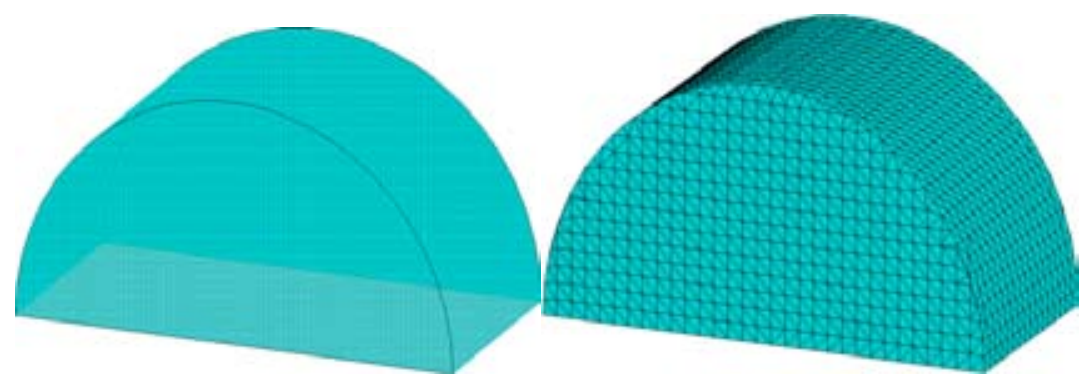

a)

b)

Рис. 1. Полигональная модель ангара $(a)$ и ее дискретизация $(b)$ 
По значениям заряда $q_{i}$ рассчитывается напряжённость $E_{i}$ для каждой заряженной сферы:

$$
\begin{aligned}
& E_{i}=\frac{q_{i}}{4 \pi \varepsilon_{0} d^{2}} \frac{q_{i}}{4 \pi \varepsilon_{0}\left(d+2 z_{i}\right)^{2}}+\sum_{j=1}^{i} \frac{q_{j}}{4 \pi \varepsilon_{0} R_{i j}^{2}}+ \\
& +\sum_{j=i+1}^{n} \frac{q_{j}}{4 \pi \varepsilon_{0} R_{i j}^{2}} \sum_{j=1}^{i} \frac{q_{j}}{4 \pi \varepsilon_{0} R_{0 i j}^{2}} \sum_{j=i+1}^{n} \frac{q_{j}}{4 \pi \varepsilon_{0} R_{0 i j}^{2}},
\end{aligned}
$$

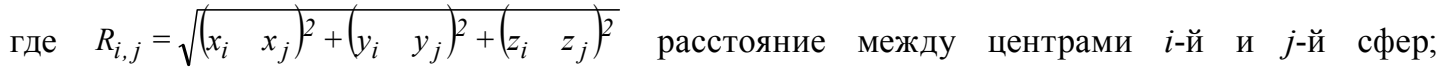

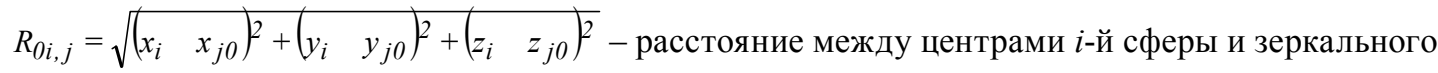
отражения $j$-й сферы.

По значению напряжённости электростатического поля в каждом узле получаем распределение напряжённости на защищаемом объекте.

Распределение напряжённости электростатического поля является основным входным параметром для расчёта траектории молниевого разряда, движущегося в сторону точки с наибольшим значением напряжённости. При этом лидер молнии испытывает постоянные возмущения в виде неоднородности электрического сопротивления воздуха, в результате чего молниевый разряд представляется в виде системы ломаных. Для воспроизведения этих ломаных в модели развития молнии используется две системы координат: глобальная и локальная. Глобальная система координат $(X, Y, Z)$ неподвижна. Начало отсчета совпадает с началом молнии. Поверхность Земли сдвинута на расстояние $H$, т. е. $(X, Y, Z+H)$. Локальная система координат $\left(x^{\prime}, y^{\prime}, z^{\prime}\right)$ перемещается вместе с молнией. Начало отсчета там, где находится лидер. Направление оси $x^{\prime}$ выбирается так, чтобы она совпадала с направлением последней ступени лидера молнии. Электрическое поле молнии на много порядков превышает суммарное поле всего остального, стримеры, окружающие лидер, как бы «нащупывают» направление движения на некотором расстоянии от лидера $L_{o p}$, а само поле молнии можно считать «источником» хаоса, которое и приводит к случайному характеру последующего продвижения лидера. Расстояние $L_{o p}$ не превышает размеров стримерной зоны, которая определяется длиной стримеров $l_{c m p}$, и зависит от линейной плотности объемного заряда $\tau$ в соответствии с соотношением [1]

$$
l_{c m p}=\frac{\tau}{4 \pi \varepsilon_{0} \quad E_{c m p}},
$$

где $E_{c m p}-$ средняя напряженность электрического поля в стримерной зоне.

В локальной системе координат направление перемещения лидера по углу $\varphi$ (рис. 2) подчиняется равномерному распределению на интервале $[0 ; \pi]$, а по углу $\alpha$ относительно оси $x^{\prime}-$ нормальному закону распределения со среднеквадратичным отклонением $\sigma_{\theta}=0.2 \div 0.3$ и больше (в зависимости от длины и мощности молнии) относительно нулевого среднего значения [1]:

$$
f_{\text {norm }}(\alpha)=\frac{1}{\sqrt{2 \pi} \sigma_{\theta}} \exp \frac{\alpha^{2}}{2 \sigma_{\theta}^{2}} .
$$

Длина $L$ отрезка молнии, «выросшей» по следу стримера, является случайной величиной с логнормальным законом распределения [2]: 


$$
f_{\text {lognorm }}(L)=\frac{1}{\sqrt{2 \pi} \sigma_{p} L} \quad \exp \quad \frac{\left(\ln \left(L / L_{0}\right)\right)^{2}}{2 \sigma_{p}^{2}} .
$$

Величины $L$ и $\tau$ выбирают из диапазона величин наблюдаемых в природе. Знак заряда выбираются случайно, исходя из условия, что 90 \% молний имеют отрицательный заряд.

В общем виде определяется направление $S$ при заданном максимальном значении направляющего вектора $E$ и угле раскрытия $\alpha[2]$ :

$$
\bar{S}=\bar{E}+\bar{d}_{1},
$$

где $\bar{d}_{1}$ - случайно отклоняющий вектор, определяемый через вектор $\bar{d}_{0}$, лежащий в плоскости, перпендикулярной вектору $\bar{E}$.

Для разряда молнии необходимо наличие как облачного, так и встречного лидера (стримера), характеризующего электрофизические процессы на поверхности земли и на объектах молниезащиты.

Модель формирования стримера схожа с моделью возникновения и развития облачного лидера молнии. Объясняется это тем, что зарождение и развитие стримера происходит практически в той же среде и в тех же условиях, что и облачного лидера. Отличительная особенность - электрофизические процессы на объектах защиты. Рассмотрим их.

Наличие достаточной ионизации воздуха вокруг объектов защиты служит одним из условий появления стримера от этих объектов. Это наблюдается, когда средняя напряженность электрического поля в пространстве между облаком и точкой начала зарождения стримера на объекте защиты не меньше средней напряженности поля в канале лидера $\left(E \geq E_{\kappa p}=3 \cdot 10^{6} \mathrm{~B} / \mathrm{M}^{2}\right)$. Развитие стримера будет наблюдаться при условии, когда электрическое поле в головке стримера достаточно для удлинения стримера на такое расстояние, при котором усиление поля будет достаточным для дальнейшего продвижения стримера и лидера молнии.

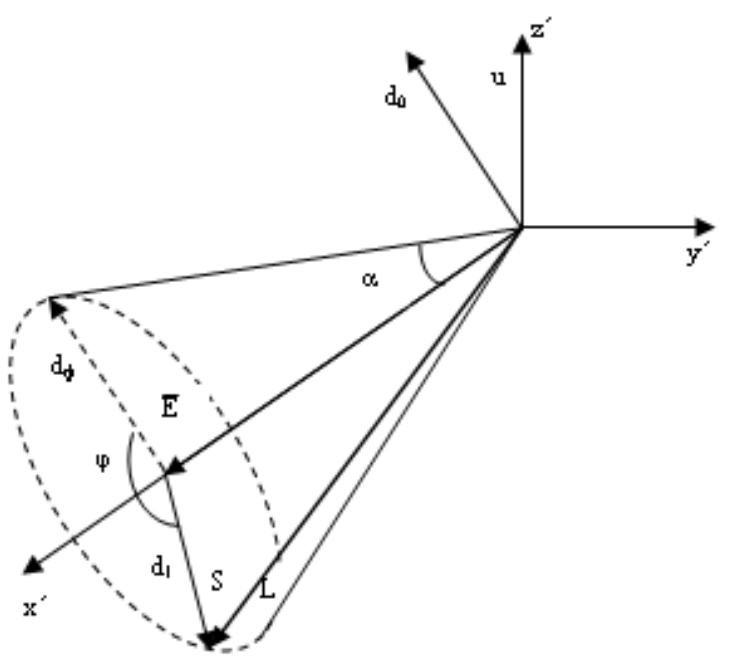

Рис. 2. Формирование ступени лидера 
Моделирование указанных условий производится на основе коррекции $q_{i}$ и $E_{i}$ с учетом зон ионизации (7), стримерной зоны (8) и проверки скорректированных значений $E_{i}$ с критическим $E_{\kappa p}$. При выполнении условия $E \geq E_{\kappa p}$, принимается решение, что ионное облако растет и есть условия для зарождения и развития стримера в узле объекта защиты, в противном случае зарождения и развития нет [2].

$$
\begin{aligned}
& R_{\text {и. } 3 i}=\sqrt{\frac{q_{i}}{4 \pi \varepsilon_{0} E_{1 i}}}, \\
& R_{\mathrm{c} .3}=\frac{q}{4 \pi \varepsilon_{0} E_{2 i} R_{0}},
\end{aligned}
$$

где $R_{\text {u.s.i }}$ - радиус зоны ионизации воздуха вокруг $i$-го узла; $E_{1 i}$ - электрическая прочность воздуха; $R_{c .3 . i}$ - радиус стримерной зоны $i$-го узла; $E_{2 i}-$ напряженность электрического поля в стримерной зоне $i$-го узла; $R_{0}$ - радиус ионного облака.

Моделирование процесса дальнейшего развития стримера производится на основе расчета: скорости роста ионного облака $V$ в условиях внешнего электрического поля $E_{0}(9)$; увеличения первоначального радиуса ионного облака $\Delta R(10)$; радиуса сферы, образованного объемным электрическим зарядом ионного облака $R_{\text {c.o. }}(11)$. В качестве радиуса ионного облака используется $R_{u . o}$, если $R_{u . o .} \leq R_{c .3 .}$ в противном случае $-R_{c .3 .}$.

$$
\begin{aligned}
& V=\mu E_{\phi}, \\
& \Delta R=\mu E_{\phi} \Delta t, \\
& R_{\text {c.o. }}=r_{0}+\Delta R,
\end{aligned}
$$

где $\mu$ - подвижность ионов; $\Delta t$ - время действия поля.

Следующим этапом моделирования является проверка выполнения условия рождения лидера внутри ионного облака. Для преодоления ионного облака необходимо, чтобы напряжение внутри облака было больше $0,6 \sqrt{R_{\text {c.o. }}}$, а в узле - больше (12):

$$
U \geq \frac{3}{2} \sqrt{\frac{\Delta U_{\min }^{4} \mu \Delta t}{2 R_{\mathrm{c} .3 .}^{2}}},
$$

где $\Delta U_{\min }$ - минимальное напряжение стримерной зоны, при которой лидер прекращает свое существование.

При выполнении указанных условий формируется первая ступень стримера. Формирование траектории движения встречного лидера осуществлялся по аналогии с формированием траектории движения облачного лидера.

Для моделирования процесса формирования последующих ступеней встречного лидера рассчитывается скорость роста встречного лидера $V_{n}(13)$, время действия внешнего электрического поля и переносимый за это время заряд (14) и ток лидера (15):

$$
\begin{aligned}
& V_{\text {л }}=a \sqrt{\Delta U_{\mathrm{r}}}, \\
& Q=\frac{i_{\mathrm{T}} R_{\mathrm{c} .3 .}}{V_{\text {л }}},
\end{aligned}
$$




$$
i_{\mathrm{T}}=\frac{2 \pi \varepsilon_{0} a \Delta U_{\mathrm{r}}^{3 / 2}}{\ln \left(\frac{1,28 E_{\mathrm{cr}}}{E_{0}}\right)},
$$

где $a$ - константа, равная $15 \mathrm{~m} / \mathrm{c} \sqrt{B} ; \Delta U_{\mathrm{r}}$-превышение потенциала головки лидера над потенциалом внешнего поля; $E_{\text {ст }}$ поле в стримерной зоне при нормальных условиях.

На основе полученных результатов определяется линейная плотность электрического заряда $\tau(16)$ и длина $L(17)$ ступени лидера:

$$
\begin{gathered}
\tau=\frac{Q}{R_{\text {с. } 3 .}}, \\
L=V_{\text {л }} \Delta t .
\end{gathered}
$$

Эти данные используются для определения координат точки на границе стримерной зоны, необходимой для задания направления формирования ступени встречного лидера.

\section{Результаты численного моделирования лидера и стримера}

на объекте молниезащиты, расчетов распределений напряженности

\section{электростатического поля и вероятности поражения объектов молниезащиты}

\section{с учетом рельефа местности}

Результаты численного моделирования облачного лидера и стримера на объектах молниезащиты представлены на рис. 3.

Используя описанную математическую модель грозового разряда и введя в нее произвольный рельеф местности, получили расчётное распределение наводимой напряжённости электростатического поля (рис. 4). Распределение не противоречит реальной действительности.

На основе выборки, состоящей из 1500 случаев численного эксперимента поражения объектов молниезащиты с учетом рельефа местности, получено расчетное распределение вероятности поражения объектов защиты (рис. 5). Анализ результатов показывает, что чем выше рельеф местности, тем выше вероятность попадания в эту местность молниевого разряда. Полученные результаты не противоречат реальной действительности. Распределения вероятно-

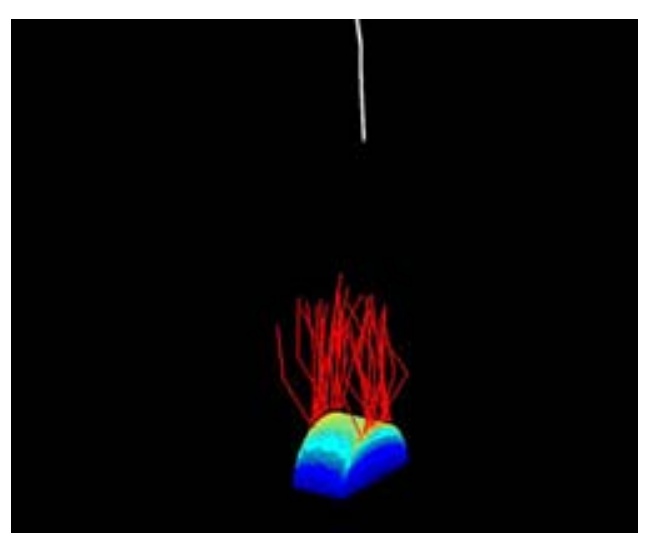

Рис. 3. Пример численного моделирования лидера и стримера на объекте молниезащиты

$$
-473-
$$




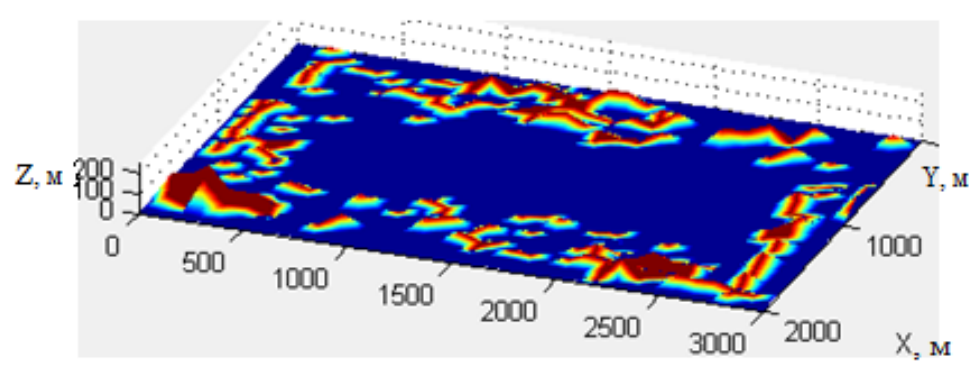

Рис. 4. Распределение напряжённости с учетом рельефа местности

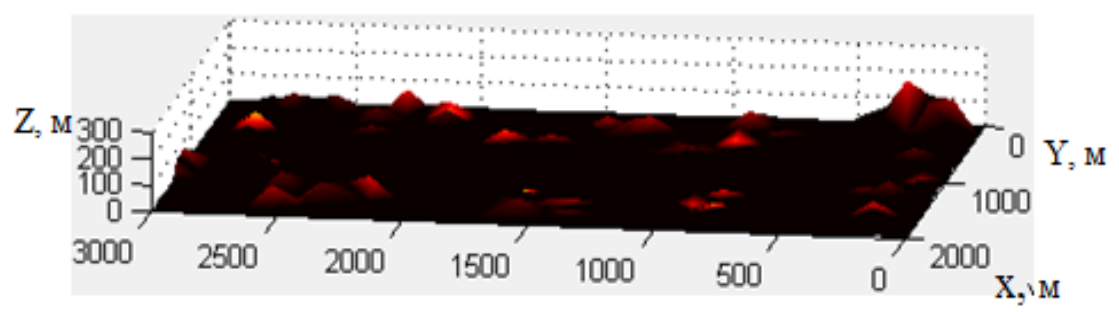

Рис. 5. Распределения вероятности поражения объектов молниезащиты

стей поражения объектов молниезащиты, расчитанные для конкретных местностей, позволят повысить эффективность молниезащиты.

\section{Выводы}

Предложена математическая модель состояния объектов молниезащиты с учетом рельефа местности. Она позволяет оптимизировать размещение средств пассивной молниезащиты объектов, а для средств активной молниезащиты - определить момент их активации и оценить эффективность их функционирования.

\section{Список литературы}

[1] Писаревский С.Ю. Моделирование и выбор оптимальных проектных решений в САПР средств молниезащчиты промышленных объектов. Дис. кан. тех. наук: Воронеж, Воронежский государственный технический университет. 2016. 161 с. [Pisarevsky S.Yu. Modeling and selection of optimal design solutions in CAD funds lightning protection of industrial facilities. Dis. can. technical Sciences: Voronezh. Voronezh state technical University, 2016, 161 p. (in Russian)]

[2] Писаревский С.Ю., Писаревский Ю.В. Имитационное моделирование процесса воздействия молнии на промышленные объекты. Электротехнические комплексы и системs ynравления: научно-технический журнал, 2009, 4, 59-64 [Pisarevsky Yu.V., Pisarevsky S.Yu. Simulation of the impact of lightning of industrial facilities. Electrical and control system: nauch.-technology, 2009, 4, 59-64 (in Russian)] 